Voix et Images

\title{
Hector Bernier, romancier de l'idéalisme abstrait
}

\section{Yvan G. Lepage}

Volume 2, numéro 3, avril 1977

Jean Éthier-Blais

URI : https://id.erudit.org/iderudit/200069ar

DOI : https://doi.org/10.7202/200069ar

Aller au sommaire du numéro

\section{Éditeur(s)}

Les Presses de l'Université du Québec

\section{ISSN}

0318-9201 (imprimé)

1705-933X (numérique)

Découvrir la revue

\section{Citer cet article}

Lepage, Y. G. (1977). Hector Bernier, romancier de l'idéalisme abstrait. Voix et Images, 2(3), 358-364. https://doi.org/10.7202/200069ar d'utilisation que vous pouvez consulter en ligne.

https://apropos.erudit.org/fr/usagers/politique-dutilisation/ 


\section{Hector Bernier, romancier de l'idéalisme abstrait}

Les préoccupations des "romanciers" québécois ont longtemps été plus politico-sociales que littéraires'. $A$ ne considérer que la pauvre production romanesque du début de notre siècle, il est relativement aisé de comprendre ce paradoxe. Exception faite de deux ou trois personnalités fortes, comme Laure Conan et Rodolphe Girard (pour ne pas mentionner Louis Hémon qui occupe une place à part dans l'histoire de la littérature québécoise), on ne voit pas que les écrivains de cette époque aient échappé à l'influence "endémique" de l'Église, elle-même alors fortement marquée par l'enseignement social de Léon XIII, d'une part, et par l'idéologie conservatrice et “unitaire» du $x x^{\theta}$ siècle, d'autre part.

Issue des collèges classiques - dont le Séminaire de Québec - dirigés par des ecclésiastiques, l'élite québécoise reçoit une formation de type humaniste fortement imprégnée de valeurs morales et abstraites, coupées, par conséquent, du réel. Cette fonction de "déracinement ${ }^{2}$ ", que remplit un tel type d'éducation, engendre des conséquences néfastes pour l'individu, dans la mesure où, tout en faisant de lui un être à part, elle l'aliène profondément.

Rejeté en marge de la société réelle, le jeune homme ainsi formé s'en trouve douloureusement et durablement affecté, partagé entre l'idéal auquel on l'a habitué à tendre de toutes ses forces et les problèmes concrets que connaît la société qui l'a vu naître et dont on l'a détaché.

On a un bon exemple de cette catégorie de jeunes gens dans ceux qui, à partir de 1904, se mettent à militer dans les cadres de l'ACJC, "croisade d'adolescents", comme l'a si bien baptisée l'abbé Groulx. Ces jeunes, épris d'idéal, "chercheront des solutions aux problèmes sociaux québécois en s'inspirant de la pensée conjuguée de Bourassa et de $M^{g r}$ L.-A. Paquet - une idéologie qui, depuis $M{ }^{9 r}$ Laflèche, associe et confond inextricablement ce qui est national, patriotique, religieux et social, dans une fusion que l'on a appelée notre idéologie «unitaire ${ }^{3}$ ".

Dans la même mouvance se créent d'autres groupes, à la fois exaspérés et généreux ${ }^{4}$ : Société du parler français (1902), Ligue nationaliste 
(1904), l'Action sociale catholique, etc. Bien que divisés sur une question de principe, comme le signale $M$. Wade ${ }^{5}$, les membres de la Ligue nationaliste et de l'ACJC n'en poursuivent pas moins les mêmes objectifs, aspirant à défendre les structures traditionnelles du Québec. "lls parlent de grandeur et rêvent d'une nation française entraînée par un chef archangélique. Leur lyrisme abstrait peut faire aujourd'hui sourire. Mais ne peuton pas voir, dans leur idéál désincarné, la forme juvénile d'un retour vers un archétype de l'inconscient collectif canadien-français ${ }^{6}$ ? "

Hector Bernier partage ces ambitions, ce «mysticisme». Son œuvre, méconnue, en témoigne on ne peut mieux.

Hector Bernier naît à Saint-Michel-de-Bellechasse, en 1886. II fait ses études classiques au Séminaire de Québec, où il a comme professeur l'abbé Camille Roy, puis ses études de droit à l'Université Laval. Devenu avocat, il écrit et publie coup sur coup deux romans ${ }^{7}$, tout en travaillant à la Bibliothèque du Parlement, à Ottawa. II s'inscrit à la Sorbonne, mais la guerre le ramène vite à Montréal où il entre à la rédaction de la Patrie. Puis il décide de s'enrôler et participe aux batailles de Courcelette et de Vimy. Gazé, il est rapatrié en 1917, mais il ne se remettra jamais complètement. II meurt d'une crise cardiaque, le 31 juillet $1947^{8}$.

\section{Au large de l'écueil}

Sur le bateau qui le ramène d'Europe, Jules, fils de l'industriel Augustin Hébert et descendant du premier colon de la Nouvelle-France, a fait la connaissance d'une Parisienne, Marguerite Delorme, venue visiter le Canada avec ses parents. Le père de Marguerite, Gilbert Delorme, milite en faveur d'un monde athée et matérialiste. C'est à l'insu de leurs parents que Jules et Marguerite se voient tous les jours, à Québec. Seule la sœur de Jules, Jeanne, est mise dans la confidence. Au soir de son élection comme député fédéral dans le comté de Salaberry, Jules, rongé par le doute, s'ouvre de son amour coupable à son curé. Ce dernier lui ordonne de rompre avec Marguerite ( $T u$ vois l'écueil, navigue au large») et l'incite à travailler à l'unité du Canada9. Augustin Hébert et Gilbert Delorme ont, chacun de son côté, eu vent des fréquentations de leurs enfants et ils les désapprouvent violemment. Séparés par la religion, Jules et Marguerite doivent se quitter. Cependant, la douleur de la séparation et les larmes rouvrent des lésions anciennes et conduisent Marguerite à une cécité incurable. Touchée par la grâce et encouragée par Jeanne, elle se rend en pèlerinage à Sainte-Anne-de-Beaupré où un miracle lui fait recouvrer la vue. Dépités, ses parents rentreront en France et Marguerite pourra épouser Jules.

\section{Ce que disait la flamme}

Fils de Gaspard Fontaine, richissime industriel, Jean est un jeune médecin animé du désir de voir prospérer sa patrie et de travailler au re- 
lèvement de la «race canadienne-française». II tente d'inculquer cet idéal à son père et à sa sœur Yvonne. Frivole et mondaine, Yvonne s'est attachée à Lucien Desloges, un "dilettante exquis" qui "n'a pas la fierté de sa race", et qu'elle finit par épouser malgré les mises en garde de Jean. Lucien ne tarde pas à se montrer sous son véritable jour et le désenchantement s'installe dans l'âme d'Yvonne. Quant à Gaspard, il n'éprouve qu'une seule "fièvre", faire de l'argent. Chacun reste donc sur ses positions jusqu'au jour où Jean décide d'épouser Lucile Bertrand, fille d'un modeste employé de Gaspard. Ce dernier s'opposant énergiquement à cette "mésalliance", Jean renonce à l'héritage, quitte le foyer paternel, épouse Lucile et se consacre aux soins des pauvres. Conquis par ce bel exemple de générosité, Gaspard et Yvonne décident de marcher sur les traces de Jean et de se dévouer pour la "race".

On ne s'étonnera pas qu'un jeune intellectuel sorti du Séminaire de Québec et qui avait vingt ans en 1906, à l'époque où les discours d'Henri Bourassa enflammaient les foules, ait donné à fond, mais à sa manière, dans l'idéologie cléricalo-nationaliste. Au large de l'écueil est dédié aux "Défenseurs de la langue française au Canada, à l'occasion du Congrès de $1912 \%$. Organisé par la Société du parler français, ce congrès ${ }^{10}$ exaltait les traditions catholiques canadiennes-françaises. De même, dans $\mathrm{Ce}$ que disait la flamme, Hector Bernier exhorte "la jeunesse canadienne [française] à cultiver, à développer dans son âme l'amour de [sa] race ${ }^{11}$ ".

Conçus dans la même atmosphère, les deux romans d'Hector Bernier présentent, à n'en pas douter, des ressemblances telles qu'on dirait deux variations sur un même thème. Les tableaux suivants le font voir:

Au large de l'écueil

Augustin Hébert, industriel

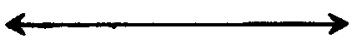
patriote (Rue des Remparts, Québec)
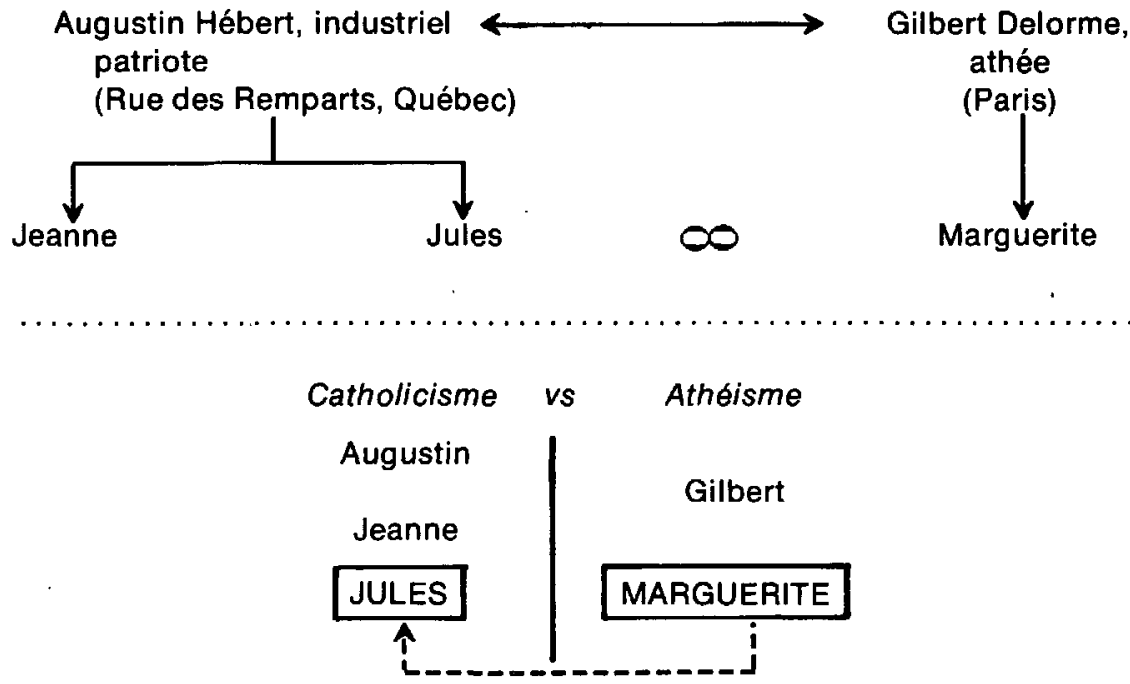


\section{Ce que disait la tlamme}
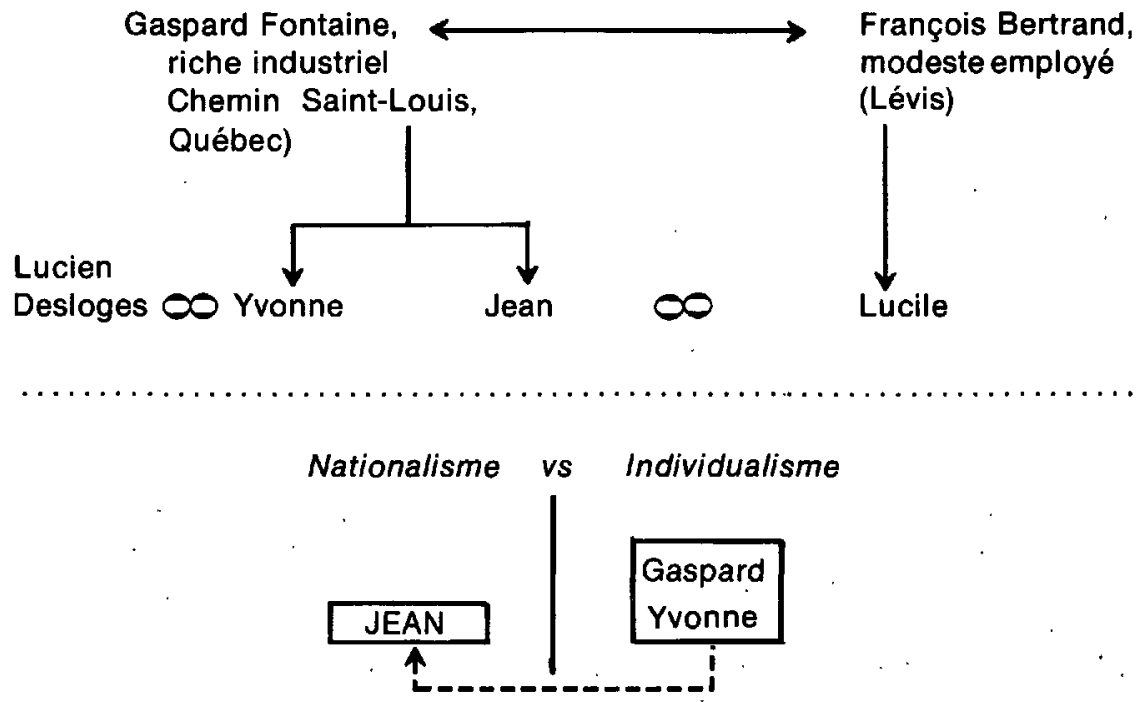

Dans Au large de l'écueil, le conflit est essentiellement religieux: catholicisme vs athéisme; il ne pourra être résorbé qu'à partir du moment où, entraînée par la ferveur patriotique et religieuse, et désillusionnée par l'athéisme matérialiste, Marguerite se convertira. Alors l'amour triomphera de l'interdit qui pesait sur le héros.

Dans $\mathrm{Ce}$ que disait la flamme, l'idéal nationaliste et la générosité de Jean s'opposent à l'individualisme et à l'égoïsme de son père et de sa scur. L'obstacle qui sépare les protagonistes disparaîtra le jour où Yvonne et Gaspard perdront les illusions qu'ils entretenaient sur l'amour ou sur la richesse et se convertiront à l'idéal de Jean.

On aura noté que le personnage principal (on peut en parler au singulier, Jean n'apparaissant, en dernière analyse, que comme une autre facette de Jules) est présenté comme un modèle et que, de ce fait, il ne saurait évoluer. Arbitrairement revêtu de vertus et de qualités idéales, le voilà désincarné, hissé au rang de symbole, de porte-parole idéologique. Plutôt qu'un héros de roman, Hector Bernier a créé un héros de western ou de bande dessinée: il ne lutte pas, il irradie.

Le récit met deux partis en présénce, celui des «bons" et celui des "méchants" dont le rôle consiste moins à se poser en antagonistes qu'à mettre le héros en valeur.

Bien que ce schéma ${ }^{12}$ se vérifie dans les deux romans, il s'applique plus parfaitement à Au large de l'écueil, où, par-delà le héros immuable, s'opposent (sans se connaître, évidemment) Augustin Hébert, représentant de 


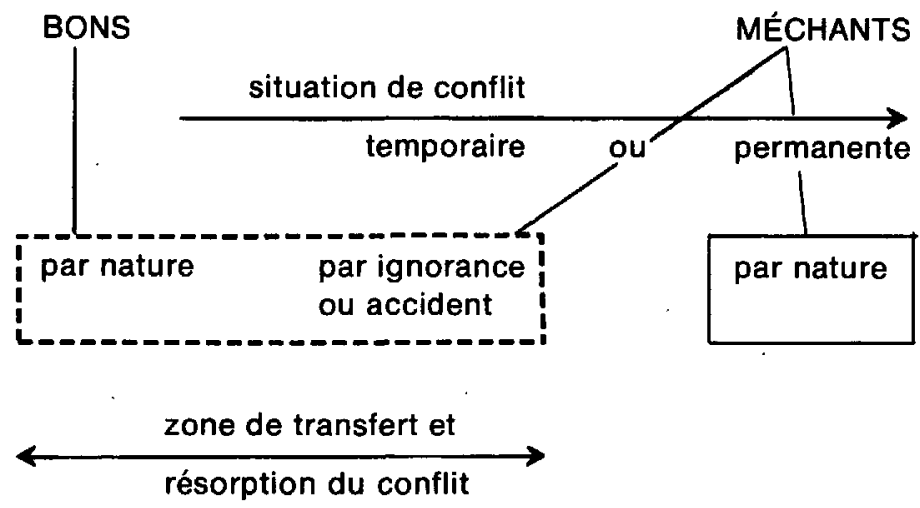

I'idéologie catholique, et Gilbert Delorme, champion de l'athéisme. II n'est pas indifférent que le conflit oppose les pères de Jules et de Marguerite, puisqu'ainsi il sanctionne l'interdit'13 dont est frappé l'amour qu'éprouvent les enfants l'un pour l'autre. Roméo et Juliette bravaient l'interdit, engendrant la tragédie. Dans Au large de l'écueil, pareille attitude serait impensable, en raison même du caractère statique du "héros". Alors intervient le deus ex machina, seule imposture capable de modifier, par miracle, la situation des amoureux, l'auteur transférant Marguerite dans le camp des "bons», comme il le fera pour Gaspard et Yvonne Fontaine dans Ce que disait la flamme. Certes, le conflit idéologique n'en est pas pour autant annulé, mais il ne concerne plus Jules et Marguerite.

Évoluant dans un univers manichéen, le jeune "héros", idéaliste à souhait, vrai parangon de vertu, a reçu une mission salvatrice - brumeuse et bien abstraite: exalter la "race canadienne-française " et la donner en modèle inaltérable au monde entier; dans une mer anglo-protestante, la langue et la culture françaises, ainsi que la religion catholique, constituent, en effet, un gage d'immutabilité. Dans ces conditions, il va de soi que les romans d'Hector Bernier n'atteignent presque jamais à une véritable intensité dramatique. Les personnages, rêveurs, s'y complaisent dans un psychologisme stérile. Même quand les dialogues occupent une place importante, comme c'est le cas dans Au large de l'écueil, ils sonnent tellement faux qu'on éprouve bien du mal à y croire; on a en effet l'impression désagréable qu'un scolastique débat contradictoire se superpose constamment aux répliques.

Les critiques les plus "lucides", ceux pour qui le roman ne saurait être mis qu'au service d'une cause littéraire, ont eu beau jeu pour éreinter Hector Bernier et démolir son œuvre avec une joie et un entrain ridiculement féroces. Ce fut le cas de Marcel Dugas ${ }^{14}$, de Jules Le Franc ${ }^{15}$ et, surtout, de Jules Fournier ${ }^{16}$. En revanche, Léon Lorrain ${ }^{17}$, Ls. D. Durand ${ }^{18}$, Edmond Léo ${ }^{19}$, Gustave Lanctôt ${ }^{20}$ et l'abbé Camille Roy ${ }^{21}$, infiniment moins sensibles à l'écriture d'Hector Bernier qu'à ses “idées", qu'ils par- 
tageaient, ont salué le beau talent du jeune auteur dans des propos qui vont de l'éloge sobre au dithyrambe le plus échevelé.

Or, cette œuvre, par ailleurs verbeuse et déclamatoire, est à mettre au compte du milieu social et intellectuel qui l'a vue naître. Elle répond en effet, d'une certaine manière, aux aspirations les plus profondes des membres de la "croisade des adolescents". Incapables de trouver au Québec l'État français catholique parfait qu'ils appellent de leurs vœux, ces jeunes le cherchent dans leurs rêves et dans leurs écrits. Et cet État idéal apparaît alors «confusément comme séparé non seulement du Canada mais de toute contingence humaine et géographique ${ }^{22}$ ». II pourrait donc y avoir homologie de structures entre cet idéalisme abstrait, «vision du monde" propre à une certaine jeunesse du début du siècle, et les romans d'Hector Bernier, où les «méchants" - qui ne le sont d'ailleurs que par accident ou ignorance - sont transmutés, sinon simplement escamotés, et où opère le charisme d'un héros immuable et «archangélique ».

En ce sens, loin d'être réactionnaire, l'œuvre d'Hector Bernier serait investie d'une réalité historique tout de même suffisante pour rendre compte, du moins en partie, de l' 'état concret de tension entre les forces d'équilibration dynamique orientées vers l'avenir et leur blocage par des forces agissant en sens contraire qui tendent à empêcher ce développement ${ }^{23}$ ” dans le Québec des années 1900.

Yvan G. Lepage Université de Moncton

1. Voir A. Lauzière, «le Roman (1860-1900)», dans P. De Grandpré, Histoire de la littérature française du Québec, t. I, Montréal, Beauchemin, 1967, chap. XxIII, p. 239, et G.-A. Vachon, «l'Espace politique et social dans le roman québécois $"$, Recherches sociographiques, vol. vII, $n^{\circ} 3(1966)$, p. 259-279.

2. R. Boily, "les Hommes politiques du Québec, 1867-1967", Revue d'histoire de l'Amérique française, numéro spécial, vol. Xxi, $n^{\circ}$ 3a (1967), p. 610.

3. J.-C. Falardeau, "Des élites traditionnelles aux élites nouvelles", Recherches sociographiques, vol. VII, $\mathrm{n}^{\circ} 1-2$ (1966) p. 136. Voir $\mathrm{L}$. Renaud, "la Fondation de I'A.C.J.C.", Idéologies au Canada français 1900-1929, Québec, PUL, 1974, p. 173-191.

4. J.-C. Falardeau, op. cit., p. 137.

5. M. Wade, les Canadiens français de 1760 a nos jours, t. 1, Montréal, Le Cercle du livre de France, 1963, p. 593.

6. J.-C. Falardeau, "Vie intellectuelle et société au début du siècle: continuité et contrastes", dans $\mathrm{P}$. De Grandpré, Histoire de la littérature trançaise du Québec, t. II, Montréal, Beauchemin, 1968, chap. I, p. 24.

7. Au large de l'écueil, Québec, Imprimerie de l'Evénement, 1912, 319 p.; Ce que disait la flamme, Québec, Imprimerie de l'Événement, 1913, xii-453 p. (préface de A.D. Decelles).

8. Voir D. Potvin, "le Romancier Hector Bernier", la Revue de I'Université Laval, vol III, $n^{\circ} 8$ (avril 1949), p. 700-706. 
9. «L'âme canadienne sera le bouclier de bronze inflexible!... Elle sera faite d'amour, amour des races fraternelles, amour de la liberté, amour du sol, tous prenant leur source en l'amour de Dieu !... Tout autant que nous, les CanadiensFrançais, les Anglais aiment le même Dieu..." "(p. 172).

“Va, mon fils, prêcher la théorie de l'âme canadienne !... [...] Tu leur diras [aux Anglais] qu'il taut oublier la haine pour ne songer qu'a l'amour, afin de former la Sainte Ligue contre l'athéisme [...]. Prêche, le génie pratique anglais fera le reste..." (p. 173).

Cette idéologie nationaliste, bien qu'épisodique, traverse le roman. Elle s'inspire de Rameau de Saint-Père et plonge ses racines jusqu'au xIxe siècle. Formulée d'abord par l'abbé Casgrain, vers 1860 (voir G.-A. Vachon, art. cité, p. 275), elle sera reprise, entre autres, par Mgr Paquet au début de notre siècle (voir P. Savard, «le Repli traditionaliste (1860-1900)», dans P. De Grandpré, op. cit., t. I, chap. $x \times 1, p$. 195). F. Ouellette a montré qu'elle survivait chez les fédéralistes québécois («les 45 ans et le pouvoir", Liberté, vol. VII, $n^{\circ} 5$ (sept.-oct. 1965), p. 396-404).

10. Voir les actes dans Premier Congrès de la langue francaise au Canada, Québec, 24-30 juin 1912, Québec, Imprimerie de l'Action sociale ltée, 1914, viii-636 p.

11. A.D. Decelles, Préface de Ce que disait la flamme, p. ix.

12. Ce schéma s'inspire de celui que $A$. Pasqualino a appliqué aux chansons de geste ( «I Reali di Francia ", Uomo e cultura, vol. III, $n^{\circ}$ 5-6 (1970) p. 87-110). Voir aussi P. Zumthor, Essai de poétique médiévale, Paris, Seuil, 1972, p. 326.

13. Voir l'article d'A. Thério, "Un cas de masochisme exemplaire: Au large de l'écueil d'Hector Bernier", Livres et auteurs québécois 1967, Montréal, Éditions Jumonville, 1968, p. 197-204.

14. L'Action. vol. II, $n^{\circ} 77$ (28 sept. 1912), p. 1.

15. L'Action, vol. IV, $\mathrm{n}^{\circ} 157$ (11 avril 1914), p. 3.

16. L'Action, vol. II, n० 70 (10 août 1912), p. 1-4 (article reproduit dans J. Fournier, Mon encrier, Montréal, Fides, “Nénuphar», 1965, p. 224-247); I'Action, vol. III, $n^{\circ} 136$ (15 nov. 1913), p. 1.

17. Le Nationaliste, vol. IX, $n^{\circ} 13$ (19 mai 1912), p. 1.

18. Le Nationaliste, vol. IX, n' 14 (26 mai 1912), p. 1 .

19. Le Devoir, vol. III, $n^{\circ} 123$ (25 mars 1912), p. 1-2; et vol. IV, $n^{\circ} 273$ (22 nov. 1913), p. 2.

20. La Patrie, vol. XXXVI, $n^{\circ} 44$ (18 avril 1914), p. 13.

21. Nouveaux Essais sur la littérature canadienne, Québec, Imprimerie de l'Action sociale Itée, 1914, p. 328-344 et 345-356.

22. J.-C. Falardeau, "Vie intellectuelle et société au début du siècle: continuité et contrastes ", dans P. De Grandpré, op. cit., t. II, chap. I, p. 24.

23. L. Goldmann, "Genèse et structure", dans Marxisme et sciences humaines, Paris, Gallimard, «Idées », p. 25. 